\title{
A Brief Overview of Multi-Scroll Chaotic Attractors Generation
}

\author{
Jinhu Lü \\ Key Laboratory of Systems and Control \\ Institute of Systems Science \\ Academy of Mathematics and Systems Science \\ Chinese Academy of Sciences \\ Beijing 100080, China \\ Email: jhlu@iss.ac.cn
}

\author{
Guanrong Chen \\ Department of Electronic Engineering \\ City University of Hong Kong \\ Hong Kong, China \\ Email: gchen@ee.cityu.edu.hk
}

\begin{abstract}
Over the last two decades, generating complex multi-scroll chaotic attractors via simple electronic circuits or simple systems has seen rapid development. This paper provides a brief overview of the subject on multi-scroll chaotic attractors generation, including some fundamental theories and design methodologies.
\end{abstract}

\section{INTRODUCTION}

Multi-scroll chaotic attractors generation has become a relatively mature research direction today, after rapid developments in the last two decades [1-14].

In retrospect, Suykens \& Vandewalle [4] firstly introduced a family of $n$-double scroll chaotic attractors by using the so-called quasi-linear function approach. Suykens et al. [5] also proposed a piecewise linear (PWL) function method for generating a complete family of $n$-scroll chaotic attractors. Aziz-Alaoui [1] presented a PWL function approach for creating multi-spiral chaotic attractors. Yalcin et al. [6] experimentally confirmed the $3-$ and 5-scroll chaotic attractors in a generalized Chua's circuit. Tang et al. [7-8] introduced a sine function to generate $n$-scroll attractors in a simple circuit with physically realizing up to a $10-$ scroll chaotic attractor observed on the oscilloscope. Similarly, Özoğuz et al. [3] suggested a nonlinear transconductor method for creating $n$-scroll chaotic attractors. Lü et al. [9] presented a switching manifold approach for generating chaotic attractors with multiple-merged basins of attraction.

In particular, Yalcin et al. [10] proposed a step circuit method for generating a family of scroll-grid chaotic attractors, including 1-D $n$-scroll, 2-D $n \times m$-grid scroll, and 3-D $n \times m \times l$-grid scroll chaotic attractors. Lü et al. [11,13] introduced a hysteresis circuit approach for creating 1-D $n$-scroll, 2-D $n \times m$-grid scroll, and 3-D $n \times m \times l$-grid scroll chaotic attractors with a rigorous theoretical proof and experimentally verifying the maximal 1-D 11-scroll, 2-D $3 \times 11$-grid scroll and 3-D $3 \times 3 \times 11$-grid scroll hysteresis chaotic attractors. Recently, Lü et al. [12-13] also initiated a saturated circuit method for generating 1-D $n$-scroll, 2-D $n \times m$-grid scroll, and 3-D $n \times m \times l$-grid scroll attractors with a rigorous mathematical proof and physically realizing the maximal 1-D 14-scroll, 2-D $14 \times 10-$ grid scroll and 3-D
$10 \times 10 \times 10-$ grid $(1000)$ scroll chaotic attractors. To that end, Yu et al. [14] suggested a novel nonlinear modulating function approach for creating $n$-scroll chaotic attractors based on a general jerk circuit.

This paper is intended to offer a very brief review, with the hope that it could benefit some new comers in both historical literature search and future research outlook. For more information, the readers are referred to our recent detailed review article [3].

The rest of this paper is organized as follows. In Section 2, a PWL function approach is introduced for generating $n$-scroll chaotic attractors. A switching manifold method and basic-circuits approach are proposed for creating multi-scroll chaotic attractors in Sections 3 and 4, respectively. In Section 5 , several other techniques for multi-scroll chaotic attractors generation are briefly discussed. Conclusions are given in Section 6.

\section{Generating N-Scroll Chaotic Attractors via PWL FUNCTIONS}

Up to now, many multi-breakpoint PWL function approaches are proposed for generating $n$-scroll chaotic attractors from Chua's circuit or other simple nonlinear systems. In the following, a generalized Chua's circuit is introduced for generating $n$-scroll chaotic attractors.

Suykens et al. [5] proposed a generalized Chua's circuit, described by

$$
\left\{\begin{array}{l}
\dot{x}=\alpha(y-h(x)) \\
\dot{y}=x-y+z \\
\dot{z}=-\beta y,
\end{array}\right.
$$

where $n$ is a natural number and $h(x)=m_{2 n-1} x+$ $\frac{1}{2} \sum_{i=1}^{2 n-1}\left(m_{i-1}-m_{i}\right)\left(\left|x+c_{i}\right|-\left|x-c_{i}\right|\right)$. System (1) is determined by the parameters set $\{\alpha, \beta, m, c\}$, where $\mathbf{m}=$ $\left(m_{0} m_{1} \cdots m_{2 n-1}\right)$ and $\mathbf{c}=\left(\begin{array}{llll}c_{1} & c_{2} & \cdots & c_{2 n-1}\end{array}\right)$.

In the case of $n$-double-scroll attractors, the components of vector $\mathbf{m}$ have alternating signs and the sign of $m_{0}$ is negative. In the case of $n$-scroll attractors with an odd number of scrolls, the components of vector $\mathbf{m}$ also have alternating signs, but the sign of $m_{0}$ is positive. 


\section{Generating Multi-Scroll Chaotic Attractors VIA SWITCHING MANIFOLD}

Lü et al. [9] introduced a systematic switching manifold approach for generating chaotic attractors with multiple merged basins of attraction from the following 3D linear controlled system:

$$
\dot{\mathbf{X}}=\mathbf{A X}+\mathbf{f}(\mathbf{X}),
$$

where $\mathbf{X}=(x, y, z)^{T}$,

$$
\mathbf{A}=\left(\begin{array}{ccc}
a & b & 0 \\
-b & a & 0 \\
0 & 0 & c
\end{array}\right)
$$

and

$$
\mathbf{f}(\mathbf{X})= \begin{cases}k\left(\begin{array}{c}
-x \\
-y \\
d
\end{array}\right), & \text { if } z+\sqrt{x^{2}+y^{2}}>k, \\
\mathbf{0}, & \text { otherwise }\end{cases}
$$

in which $a, b, c, d, k$ are real parameters. System (2) with controller $\mathbf{f}(\mathbf{X})$ can create chaotic attractors within a wide range of parameter values.

To generate a chaotic attractor with two merged basins of attraction, one can modify the controller $\mathbf{f}(\mathbf{X})$ as follows:

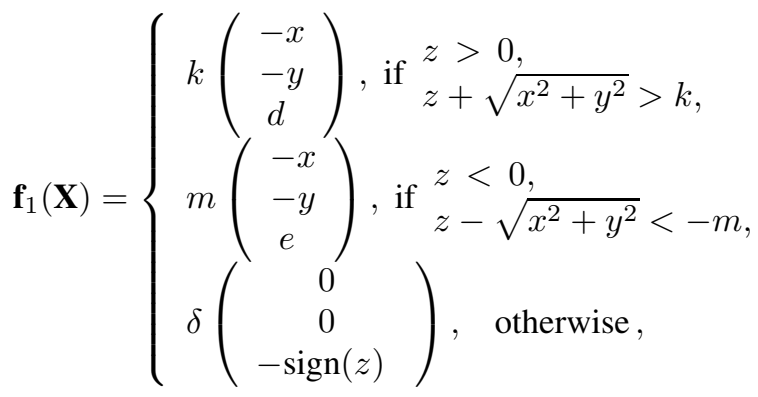

where $a, b, c, d, e, k, m, \delta$ are all real parameters.

Similarly, one can create chaotic attractors with $n$ merged basins of attraction. The formalized design approach is outlined as follows:

(i) Partition the whole space into $n$ subspaces. For convenience, one may partition the space along the $z$-axis.

(ii) Duplicate the original attractors, the upper-attractor and the lower-attractor, to every subspace.

(iii) Use the switching controller to connect all the $n$ independent attractors, so as to form a single chaotic attractor with multiple merged basins of attraction.

Here, the switching controller can be chosen as $\delta \operatorname{sign}(z-$ $h_{i}$ ), where the height $h_{i}$ (between two neighboring subspaces) should be smaller than the height of a single chaotic attractor.

\section{Generating Multi-Directional Multi-Scroll ChaOtic ATtractors Via Basic Circuits}

This section reviews several approaches for generating multi-directional multi-scroll chaotic attractors by using three basic circuits: step circuit, hysteresis circuit and saturated circuit.

\section{A. Step circuit}

Yalcin et al. [10] proposed a new family of scroll and grid-scroll attractors by using the step circuit, including 1-D $n$-scroll, 2-D $n \times m$-grid scroll, and 3-D $n \times m \times l$-grid scroll chaotic attractors. The state equation of this family of systems is given by

$$
\dot{\mathbf{X}}=\mathbf{A X}+\mathbf{B} \sigma(\mathbf{C X})
$$

where $\mathbf{X}=(x, y, z)^{T}, \mathbf{B}=\operatorname{diag}\{1,1,1\}$ and

$$
\mathbf{A}=\left(\begin{array}{ccc}
0 & 1 & 0 \\
0 & 0 & 1 \\
-a & -a & -a
\end{array}\right), \quad \mathbf{C}=\left(\begin{array}{lll}
0 & 1 & 0 \\
0 & 0 & 1 \\
1 & 0 & 0
\end{array}\right)
$$

There are three different cases:

(i) 1-D $n$-scroll chaotic attractors:

$$
\left\{\begin{array}{l}
b_{y}=b_{z}=0 \\
\sigma(\cdot)=\left(\begin{array}{c}
0 \\
0 \\
f_{1}(\cdot)
\end{array}\right)
\end{array}\right.
$$

where $f_{1}(x)=\sum_{i=1}^{M_{x}} g_{\frac{-2 i+1}{2}}(x)+\sum_{i=1}^{N_{x}} g_{\frac{2 i-1}{2}}(x)$ and

$$
g_{\theta}(\zeta)=\left\{\begin{array}{c}
1, \quad \zeta \geq \theta \quad \theta>0 \\
0, \quad \zeta<\theta \quad \theta>0 \\
0, \zeta \geq \theta \quad \theta<0 \\
-1, \zeta<\theta \quad \theta<0
\end{array}\right.
$$

which belongs to the sector $[0,2]$.

(ii) 2-D $n \times m$-grid scroll chaotic attractors:

$$
\left\{\begin{array}{c}
b_{y}=-1, b_{z}=0 \\
\sigma(\cdot)=\left(\begin{array}{c}
f_{1}(\cdot) \\
0 \\
f_{2}(\cdot)
\end{array}\right)
\end{array}\right.
$$

where $f_{1}(\cdot)$ is defined in $(i), f_{2}(x)=\sum_{i=1}^{m-1} \beta g_{p_{i}}(x)$ with $p_{i}=M_{y}+0.5+(i-1)\left(M_{y}+N_{y}+1\right), \beta=M_{y}+N_{y}+1$, which belongs to the sector $\left[0, \frac{M_{y}+N_{y}+1}{M_{y}+0.5}\right]$.

(iii) 3-D $n \times m \times l$-grid scroll chaotic attractors:

$$
\left\{\begin{array}{l}
b_{y}=-1, b_{z}=-1 \\
\sigma(\cdot)=\left(\begin{array}{l}
f_{1}(\cdot) \\
f_{1}(\cdot) \\
f_{3}(\cdot)
\end{array}\right)
\end{array}\right.
$$

where $f_{1}(\cdot)$ is defined in $(i), f_{3}(x)=\sum_{i=1}^{k-1} \gamma g_{n_{l}}(x)$ with $n_{l}=\rho+0.5+(l-1)(\rho+\zeta+1), \gamma=\rho+\zeta+1$, $\rho=\left|\min _{i, j}\left\{u_{i}^{e q, y}+u_{j}^{e q, z}\right\}\right|, \zeta=\left|\max _{i, j}\left\{u_{i}^{e q, y}+u_{j}^{e q, z}\right\}\right|$, in which $u_{i}^{e q, y}, u_{j}^{e q, z}$ are the vectors for the $y$ and $z$ variables related to the equilibrium points, respectively, which belongs to the sector $\left[0, \frac{\zeta+\rho+1}{\rho+0.5}\right]$. 


\section{B. Hysteresis circuit}

Lü et al. [11] introduced a new systematic approach for generating multi-directional multi-scroll chaotic attractors from a 3D linear autonomous system using hysteresis series, including 1-D $n$-scroll, 2-D $n \times m$-grid scroll, and 3-D $n \times m \times$ $l$-grid scroll attractors. The system is described by (3) with $\mathbf{B}=-\mathbf{A}, \mathbf{C}=\operatorname{diag}\{1,1,1\}$, and

$$
\mathbf{A}=\left(\begin{array}{ccc}
0 & 1 & 0 \\
0 & 0 & 1 \\
-a & -b & -c
\end{array}\right)
$$

There are three different cases to consider:

(i) 1-D hysteresis $n$-scroll chaotic attractors:

$$
\sigma(\mathbf{X})=\left(\begin{array}{c}
h\left(x, p_{1}, q_{1}\right) \\
0 \\
0
\end{array}\right),
$$

where the hysteresis series function $h\left(x, p_{1}, q_{1}\right)$ is defined by

$$
h\left(x, p_{1}, q_{1}\right)=\left\{\begin{array}{lll}
-p_{1} & \text { if } & x<-p_{1}+1 \\
i & \text { if } & i-1<x<i+1 \\
q_{1} & \text { if } & x>p_{1}-1 .
\end{array}\right.
$$

(ii) 2-D hysteresis $n \times m$-grid scroll chaotic attractors:

$$
\sigma(\mathbf{X})=\left(\begin{array}{c}
h\left(x, p_{1}, q_{1}\right) \\
h\left(y, p_{2}, q_{2}\right) \\
0
\end{array}\right)
$$

where the hysteresis series functions $h\left(x, p_{1}, q_{1}\right)$ and $h\left(y, p_{2}, q_{2}\right)$ are similarly defined by (4).

(iii) 3-D hysteresis $n \times m \times l$-grid scroll chaotic attractors:

$$
\sigma(\mathbf{X})=\left(\begin{array}{c}
h\left(x, p_{1}, q_{1}\right) \\
h\left(y, p_{2}, q_{2}\right) \\
h\left(z, p_{3}, q_{3}\right)
\end{array}\right)
$$

where the hysteresis series functions $h\left(x, p_{1}, q_{1}\right)$, $h\left(y, p_{2}, q_{2}\right)$, and $h\left(z, p_{3}, q_{3}\right)$ are similarly defined by (4).

Lü et al. [13] also designed a novel block circuit diagram for physically realizing 1-D $5 \sim 11$-scroll, 2-D $3 \times 5 \sim$ 11-grid scroll, and 3-D $3 \times 3 \times 5 \sim 11$-grid scroll chaotic attractors.

\section{Saturated circuit}

Lü et al. [12] initiated a 3D saturated multi-scroll chaotic system described by (3) with $\mathbf{C}=\operatorname{diag}\{1,1,1\}$ and

$$
\mathbf{A}=\left(\begin{array}{ccc}
0 & 1 & 0 \\
0 & 0 & 1 \\
-a & -b & -c
\end{array}\right), \quad \mathbf{B}=\left(\begin{array}{ccc}
0 & -\frac{d_{2}}{b} & 0 \\
0 & 0 & -\frac{d_{3}}{c} \\
d_{1} & d_{2} & d_{3}
\end{array}\right)
$$

This system can produce three different types of attractors, as follows:

(i) 1-D saturated $n$-scroll chaotic attractors:

$$
\sigma(\mathbf{C X})=\left(\begin{array}{c}
f\left(x ; k_{1}, h_{1}, p_{1}, q_{1}\right) \\
0 \\
0
\end{array}\right),
$$

where the saturated function series $f\left(x ; k_{1}, h_{1}, p_{1}, q_{1}\right)$ is defined by

$$
\begin{aligned}
& f\left(x ; k_{1}, h_{1}, p_{1}, q_{1}\right)= \\
& \begin{cases}\left(2 q_{1}+1\right) k_{1}, & \text { if } x>q_{1} h_{1}+1 \\
k_{1}\left(x-i h_{1}\right)+2 i k_{1}, & \text { if } \quad-p_{1} \leq i \leq h_{1} \mid \leq 1 \\
& \text { if } 1<x-i h_{1}<h_{1}-1 \\
(2 i+1) k_{1}, & \text { if } x<-p_{1} \leq i \leq q_{1}-1 \\
-\left(2 p_{1}+1\right) k_{1}, & \text { if }-1 .\end{cases}
\end{aligned}
$$

(ii) 2-D saturated $n \times m$-grid scroll chaotic attractors:

$$
\sigma(\mathbf{C X})=\left(\begin{array}{c}
f\left(x ; k_{1}, h_{1}, p_{1}, q_{1}\right) \\
f\left(y ; k_{2}, h_{2}, p_{2}, q_{2}\right) \\
0
\end{array}\right),
$$

where $f\left(x ; k_{1}, h_{1}, p_{1}, q_{1}\right)$ and $f\left(y ; k_{2}, h_{2}, p_{2}, q_{2}\right)$ are similarly defined by (5).

(iii) 3-D saturated $n \times m \times l$-grid scroll chaotic attractors:

$$
\sigma(\mathbf{C X})=\left(\begin{array}{c}
f\left(x ; k_{1}, h_{1}, p_{1}, q_{1}\right) \\
f\left(y ; k_{2}, h_{2}, p_{2}, q_{2}\right) \\
f\left(z ; k_{3}, h_{3}, p_{3}, q_{3}\right)
\end{array}\right),
$$

where $f\left(x ; k_{1}, h_{1}, p_{1}, q_{1}\right), \quad f\left(y ; k_{2}, h_{2}, p_{2}, q_{2}\right), \quad$ and $f\left(z ; k_{3}, h_{3}, p_{3}, q_{3}\right)$ are similarly defined by (5).

Moreover, Lü et al. [13] also designed a block circuitry for experimentally verifying 1-D 10,12, 14-scroll, 2D $10,12,14 \times 10-$ grid scroll, and 3 -D $10 \times 10 \times 10-$ grid scroll chaotic attractors. Particularly, it was the first time in the literature to report the experimental verification of 1-D 14-scroll, 2-D $14 \times 10$-grid scroll and 3-D $10 \times 10 \times$ 10-grid (1000) scroll chaotic attractors.

\section{Generating Multi-Scroll Chaotic Attractors VIA OTHER APPROACHES}

In this section, several other techniques for creating multiscroll chaotic attractors are briefly discussed.

\section{A. Sine function approach}

Tang et al. [7] applied the sine function to replace the nonlinear characteristic function of Chua's circuit and obtained a modified Chua's circuit as follows:

$$
\left\{\begin{array}{l}
\dot{x}=\alpha(y-f(x)) \\
\dot{y}=x-y+z \\
\dot{z}=-\beta y
\end{array}\right.
$$

where

$$
f(x)= \begin{cases}\frac{b \pi}{2 a}(x-2 a c) & x \geq 2 a c \\ -b \sin \left(\frac{\pi x}{2 a}+d\right) & -2 a c<x<2 a c \\ \frac{b \pi}{2 a}(x+2 a c) & x \leq-2 a c\end{cases}
$$

and

$$
d= \begin{cases}\pi & \text { for } c \text { is even } \\ 0 & \text { for } c \text { is odd },\end{cases}
$$

in which $\alpha, \beta, a, b, c, d$ are real parameters.

Note that system (6) can generate $(c+1)$-scroll chaotic attractors. Tang et al. [7] also constructed an electronic circuit to experimental verify these $n$-scroll chaotic attractors. 


\section{B. Transconductor function method}

Similarly, Özoğuz et al. [3] constructed an $n$-scroll chaotic attractors generator by using the smooth hyperbolic tangent functions, which is described by

$\left(\begin{array}{c}\dot{x} \\ \dot{y} \\ \dot{z}\end{array}\right)=\left(\begin{array}{ccc}0 & 1 & 0 \\ 0 & 0 & 1 \\ 0 & -a & -a\end{array}\right)\left(\begin{array}{l}x \\ y \\ z\end{array}\right)+\left(\begin{array}{c}0 \\ 0 \\ -a f\left(x_{1}\right)\end{array}\right)$,

where $f(x)=\sum_{j=-N}^{M}(-1)^{j-1} \tanh k\left(x-o_{j}\right)$ and $M, N$ are odd integers. System (7) can generate a $\frac{M+N+2}{2}-$ scroll chaotic attractor.

\section{General nonlinear modulating functions approach}

The general jerk circuit is described by

$$
\dddot{x}+\beta \ddot{x}+\gamma \dot{x}=f(x),
$$

where $\beta, \gamma$ are real parameters, $f(x)$ is a nonlinear function, $\dot{x}=\frac{d x}{d \tau}$ is the velocity, $\ddot{x}=\frac{d^{2} x}{d \tau^{2}}$ is the acceleration, and $\dddot{x}=\frac{d^{3} x}{d \tau^{3}}$ is the jerk.

Yu et al. [14] introduced a PWL function with varying breakpoints and slopes to create $n$-scroll chaotic attractors from (8). It includes two cases with even and odd numbers of scrolls, respectively:

(1) Even number of scrolls:

$$
\begin{aligned}
f_{1}(x)= & \sum_{n=-M}^{M} \frac{A}{2 \alpha_{n}}\left[\left|\left(x-\frac{2 A n}{B}\right)+\alpha_{n}\right|\right. \\
& \left.-\left|\left(x-\frac{2 A n}{B}\right)-\alpha_{n}\right|\right]-B x,
\end{aligned}
$$

where parameters $A>0,0.8 \leq B \leq 1.2, \alpha_{n} \in$ $\left(0, \frac{3 A}{10 B}\right](n=0, \pm 1, \cdots, \pm M), M=1,2, \cdots$, which can create $2 M+2$ scrolls in the chaotic attractor.

(2) Odd number of scrolls:

$$
\begin{aligned}
f_{2}(x)= & \sum_{\substack{n=-M \\
n \neq 0}}^{M} \frac{A}{2 \alpha_{n}}\left[\left|\left(x-\frac{A}{B}\left(2 n-\frac{|n|}{n}\right)\right)+\alpha_{n}\right|\right. \\
& \left.-\left|\left(x-\frac{A}{B}\left(2 n-\frac{|n|}{n}\right)\right)-\alpha_{n}\right|\right]-B x,
\end{aligned}
$$

where parameters $A>0,0.8 \leq B \leq 1.2, \alpha_{n} \in$ $\left(0, \frac{3 A}{10 B}\right](n= \pm 1, \pm 2, \cdots, \pm M), M=1,2, \cdots$, which can create $2 M+1$ scrolls in the chaotic attractor.

D. Hyperchaotic multi-scroll attractors from the modified MCK circuit

Yalcin et al. [2] proposed a modified MCK circuit method for creating $n$-double scroll hyperchaotic attractors, which is given by

$$
\left\{\begin{array}{l}
\dot{x}=\alpha(g(y-x)-z) \\
\dot{y}=\beta(-g(y-x)-w) \\
\dot{z}=x+z \\
\dot{w}=\gamma y
\end{array}\right.
$$

where $g(y-x)=m_{0}(y-x)+\frac{1}{2} \sum_{i=1}^{2 n-1}\left(m_{i}-m_{i-1}\right)(\mid y-$ $\left.x+c_{i}|-| y-x-c_{i} \mid\right)$.
Moreover, Yu et al. [3] further modified its PWL characteristic to generate $n$-scroll hyperchaotic attractors, as follows: $g(y-x)=m_{N-1}(y-x)+\frac{1}{2} \sum_{i=1}^{N-1}\left(m_{i-1}-m_{i}\right)(\mid y-x+$ $\left.c_{i}|-| y-x-c_{i} \mid\right)$.

\section{CONClusions}

This paper briefly reviews the main advances of multiscroll chaotic attractors generation, including PWL method, switching manifold approach, basic circuits method, sine and transconductor functions techniques, nonlinear modulating functions approach, and modified MCK circuit method. A more detailed review can be found in our overview article [3].

\section{ACKNOWLEDGMENTS}

This work was supported by the National Natural Science Foundation of China under Grants No.60304017 and No.20336040, and the Scientific Research Startup Special Foundation on Excellent PhD Thesis and Presidential Award of the Chinese Academy of Sciences.

\section{REFERENCES}

[1] M. A. Aziz-Alaoui, "Differential equations with multispiral attractors," Int. J. Bifurcation Chaos, vol. 9, no. 6, pp. 1009-1039, Jun. 1999.

[2] M. E. Yalcin, J. A. K. Suykens, and J. P. L. Vandewalle, Cellular Neural Networks, Multi-Scroll Chaos and Synchronization. Singapore: World Scientific, 2005.

[3] J. Lü and G. Chen, "Multi-scroll chaos generation: Theories, methods and applications," Int. J. Bifurcation Chaos, vol. 16, in press, 2006.

[4] J. A. K. Suykens and J. Vandewalle, "Generation of $n$-double scrolls ( $n=1,2,3,4, \cdots)$," IEEE Trans. Circuits Syst. I, vol. 40, no. 11, pp. 861-867, Nov. 1993.

[5] J. A. K. Suykens, A. Huang, and L. O. Chua, "A family of $n$-scroll attractors from a generalized Chua's circuit," Int. J. Electron. Commun., vol. 51, no. 3, pp. 131-138, May 1997.

[6] M. E. Yalcin, J. A. K. Suykens, and J. Vandewalle, "Experimental confirmation of 3- and 5-scroll attractors from a generalized Chua's circuit," IEEE Trans. Circuits Syst. I vol.47, no. 3, pp. 425-429, May 2000 .

[7] K. S. Tang, G. Q. Zhong, G. Chen, and K. F. Man, "Generation of $n$-scroll attractors via sine function," IEEE Trans. Circuits Syst. I, vol. 48 , no. 11 , pp. $1369-1372$, Nov. 2001.

[8] G. Zhong, K. F. Man, and G. Chen, "A systematic approach to generating $n$-scroll attractors," Int. J. Bifurcation Chaos, vol. 12, no. 12, pp. $2907-$ 2915, Dec. 2002.

[9] J. Lü, X. Yu, and G. Chen, "Generating chaotic attractors with multiple merged basins of attraction: A switching piecewise-linear control approach," IEEE Trans. Circuits Syst. I, vol. 50, no. 2, pp. 198-207, Feb. 2003.

[10] M. E. Yalcin, J. A. K. Suykens, J. Vandewalle, and S. Ozoguz, "Families of scroll grid attractors," Int. J. Bifurcation Chaos, vol. 12, no. 1, pp. 23-41, Jan. 2002

[11] J. Lü, F. Han, X. Yu, and G. Chen, "Generating 3-D multi-scroll chaotic attractors: A hysteresis series switching method," Automatica, vol. 40, no. 10, pp. 1677-1687, Oct. 2004.

[12] J. Lü, G. Chen, X. Yu, and H. Leung, "Design and analysis of multiscroll chaotic attractors from saturated function series," IEEE Trans. Circuits Syst. I, vol. 51, no. 12, pp. 2476-2490, Dec. 2004.

[13] J. Lü, S. M. Yu, H. Leung, and G. Chen, "Experimental verification of multi-directional multi-scroll chaotic attractors," IEEE Trans. Circuits Syst. I, vol. 53, no. 1, pp. 149-165, Jan. 2006.

[14] S. M. Yu, J. Lü, H. Leung, and G. Chen, "Design and implementation of $n$-scroll chaotic attractors from a general jerk circuit," IEEE Trans. Circuits Syst. I, vol. 52, no. 7, pp. 1459-1476, Jul. 2005. 